\title{
Baicalin and baicalein attenuate renal fibrosis in vitro via inhibition of the TGF- $\beta 1$ signaling pathway
}

\author{
QIN HU ${ }^{1,2}$, LINA GAO ${ }^{1}$, BO PENG $^{3}$ and XINMIN LIU ${ }^{2}$ \\ ${ }^{1}$ College of Life Sciences and Bio-Engineering, Beijing University of Technology, Beijing 100024; \\ ${ }^{2}$ Institute of Medicinal Plant Development, Chinese Academy of Medical Sciences, Beijing 100193; \\ ${ }^{3}$ Institute of Chinese Materia Medica, Chinese Academy of Medical Sciences, Beijing 100700, P.R. China
}

Received October 2, 2016; Accepted June 5, 2017

DOI: $10.3892 /$ etm.2017.4888

\begin{abstract}
Baicalin and baicalein are flavonoid compounds derived from Scutellaria baicalensis Georgi. These compounds have been used in the treatment of numerous diseases, including fibrotic diseases. However, research regarding their antifibrotic effects and mechanism of action in renal fibrosis is limited. In the present study, normal rat kidney interstitial fibroblast (NRK-49F) cells were stimulated with transforming growth factor (TGF)- $\beta 1$, with or without baicalin/baicalein, and assessed for proliferation, apoptosis, extracellular matrix (ECM) accumulation, collagen expression, TGF- $\beta 1$ expression and mothers against decapentaplegic homolog 3 (SMAD3) protein activation. The results revealed that baicalin and baicalein exhibited antifibrotic effects in vitro, whereas baicalein had a stronger inhibitory action compared with baicalin on TGF- $\beta 1$-induced NRK-49F cell proliferation, deposition of ECM, collagen synthesis, endogenous TGF- $\beta 1$ expression and phosphorylation of SMAD3. In conclusion, the findings of the present study indicate that baicalin and baicalein, particularly baicalein, exhibit antifibrotic effects in vitro by inhibiting the TGF- $\beta 1$ pathway. Therefore, these compounds have the potential to be developed as novel agents to treat renal fibrosis.
\end{abstract}

\section{Introduction}

Renal fibrosis is a common pathological effect of chronic renal injury and its progression leads to renal failure. Despite extensive research, the etiology and pathogenesis of renal fibrosis remain unclear, and an effective therapy with limited side effects is still lacking. Previous studies have demonstrated that excessive fibrogenesis is primarily due to hyperfunctioning fibroblasts $(1,2)$. When fibroblasts remain active for a prolonged

Correspondence to: Professor Xinmin Liu, Institute of Medicinal Plant Development, Chinese Academy of Medical Sciences, 151 Malianwa North Road, Beijing 100193, P.R. China

E-mail: liuxinmin@hotmail.com

Key words: renal fibrosis, baicalin, baicalein, transforming growth factor $\beta 1$ period of time this results in elevated collagen secretion and fibroproliferative disorders (3). Transforming growth factor (TGF)- $\beta$, a cytokine that is involved in cell differentiation, proliferation and apoptosis, has been demonstrated to serve an essential role in this fibrotic process (4).

Baicalin and its aglycone baicalein are the primary flavones found in the dry roots of the Chinese herb Scutellaria baicalensis Georgi. Previous studies have demonstrated the multiple functions of baicalin and baicalein, including anxiolytic, antioxidant, antitumor, anti-inflammatory and anti-infection effects $(5,6)$. These compounds have been studied as potential agents for the treatment of allergic diseases (7), inflammatory diseases $(8,9)$, cardiovascular disorders (10), malignancies (11) and ocular diseases (12).

Baicalin and baicalein have traditionally been used to treat cardiovascular and liver diseases in Asia, and have demonstrated certain therapeutic effects on hepatic fibrosis, cardiac fibrosis, pulmonary fibrosis and renal interstitial fibrosis $(13,14)$. The attenuation of fibrosis by baicalin and baicalein was partially attributed to the suppression of collagen I and fibronectin expression (15), inhibition of the proliferation and induction of apoptosis of fibroblasts (16), inhibition of the infiltration of macrophages and lymphocytes, and the suppression of differentiation of Th17 cells (17). Previously, our group reported the antifibrotic activity of baicalin and baicalein, which was detected by high-throughput screening in an in vitro model (18). The present study aimed to further investigate the antifibrotic effects of baicalin and baicalein, in addition to the underlying molecular mechanisms of these effects.

\section{Materials and methods}

TGF- $\beta 1$-induced in vitro model of fibrosis. Normal rat kidney interstitial fibroblast (NRK-49F) cells were obtained from ATCC (Manassas, VA, USA) and cultured in Dulbecco's modified Eagle's medium (Gibco; Thermo Fisher Scientific, Inc., Waltham, MA, USA) supplemented with $100 \mathrm{U} / \mathrm{ml}$ penicillin $\mathrm{G}, 100 \mu \mathrm{g} / \mathrm{ml}$ streptomycin and $10 \%$ fetal bovine serum (Gibco; Thermo Fisher Scientific, Inc.). The TGF- $\beta 1$-induced in vitro fibrosis model was generated as described previously (13). Briefly, NRK-49F cells were seeded in collagen I-coated 96-well plates at a density of 5,000 cells per well and 
cultured at $37^{\circ} \mathrm{C}$ with $5 \% \mathrm{CO}_{2}$ for $72 \mathrm{~h}$, after which the medium was replaced with serum-free medium. After a further incubation for $48 \mathrm{~h}$, recombinant human TGF- $\beta 1(5 \mathrm{ng} / \mathrm{ml})$ was added with or without baicalin/baicalein $(20,40,80 \mu \mathrm{M}$; National Institute for the Control of Pharmaceutical and Biological Products, Beijing, China) and the cells were incubated for an additional $48 \mathrm{~h}$. The cells were then fixed in methanol overnight at $-20^{\circ} \mathrm{C}$ and stained with a $0.1 \%$ Picrosirius red (PSR; Sigma-Aldrich; Merck KGaA, Darmstadt, Germany) at room temperature for $4 \mathrm{~h}$. The staining solution was removed and the cells were washed with $0.1 \%$ acetic acid, followed by the addition of $0.1 \mathrm{~N}$ sodium hydroxide. Antifibrotic activity was then assessed by measuring the optical density (OD) at $540 \mathrm{~nm}$.

Cell viability assays. NRK-49F cells were seeded into a 96-well plate at a density of $5 \times 10^{4}$ cells/well. Once the cells reached $80 \%$ confluence, the medium was replaced with serum-free medium. After an additional incubation for $48 \mathrm{~h}$, the cells were treated with TGF- $\beta 1(5 \mathrm{ng} / \mathrm{ml})$ and baicalin/baicalein $(20,40$, $80 \mu \mathrm{M}$ ) for 2 days. Cell viability was measured using the MTS and 5-bromo-2-deoxyuridine (BrdU) incorporation assays. For the MTS assay, $20 \mu \mathrm{l}$ of MTS solution $(5 \mathrm{mg} / \mathrm{ml}$ ) was added to each well and the plate was incubated at $37^{\circ} \mathrm{C}$ for $4 \mathrm{~h}$. The absorbance of the wells was then recorded at $490 \mathrm{~nm}$. For the BrdU incorporation assay, the medium was removed and replaced with medium containing $10 \mu \mathrm{M}$ BrdU. After incubation for $14 \mathrm{~h}$, the cells were washed with PBS and fixed with $4 \%$ paraformaldehyde at room temperature for $1 \mathrm{~h}$. The DNA was denatured with $4 \mathrm{M} \mathrm{HCl}$ for $10 \mathrm{~min}$ at room temperature and the plate was blocked with $5 \%$ goat serum (Thermo Fisher Scientific, Inc.) containing 0.05\% Triton X-100. BrdU incorporation was detected by incubation with a primary mouse monoclonal anti-BrdU antibody (1:100, B-2531; Merck $\mathrm{KGaA}$ ) at $4^{\circ} \mathrm{C}$ overnight and an Alexa Fluor 555-labeled goat anti-mouse immunoglobulin $\mathrm{G}(\mathrm{IgG})$ secondary antibody (1:500, A021422; Thermo Fisher Scientific, Inc.) at $37^{\circ} \mathrm{C}$ for 30 min. Cell nuclei were counterstained with DAPI (Merck $\mathrm{KGaA}$ ) at room temperature for $10 \mathrm{~min}$. BrdU-positive cells were counted under a fluorescence microscope using ImageJ software (version 2.1; National Institutes of Health, Bethesda, MD, USA).

Apoptosis assay. Apoptotic cells were detected using the Dead Cell apoptosis kit with Annexin V Alexa Fluor 488/propidium iodide (PI) (Thermo Fisher Scientific, Inc.). Briefly, NRK-49F cells were harvested by centrifugation at $800 \mathrm{x}$ g (room temperature for $5 \mathrm{~min}$ ) and the cell pellet was resuspended in Annexin V binding buffer at room temperature. After a 30 min incubation in the dark, the cells were washed with a buffer for fluorescence-activated cell sorting and incubated with PI solution prior to flow cytometric detection. Early (Annexin V-positive, PI-negative) and late (Annexin V-positive, PI-positive) apoptotic cells were analyzed using the WinMDI software version 2.9 (The Scripps Research Institute, La Jolla, CA, USA).

Immunofluorescence assay. Immunofluorescence staining was performed to detect the expression of mothers against decapentaplegic homolog 3 (SMAD3) protein and $\alpha$-smooth muscle actin ( $\alpha$-SMA). Briefly, NRK-49F cells were fixed with $4 \%$ paraformaldehyde at room temperature for $15 \mathrm{~min}$ and permeabilized for 5 min using $0.1 \%$ Triton X-100. After washing with PBS, the cells were blocked with $5 \%$ bovine serum albumin (Merck KGaA) in PBS containing Tween-20 at room temperature for $1 \mathrm{~h}$, then incubated with primary mouse anti- $\alpha$-SMA (1:400, A5228; Merck KGaA) and rabbit anti-phosphorylated-SMAD3 (1:200, ab52903; Abcam, Cambridge, MA, USA) antibodies overnight at $4^{\circ} \mathrm{C}$. Subsequently, the cells were incubated with Alexa Fluor 555-conjugated anti-mouse IgG (1:500, A021422; Thermo Fisher Scientific, Inc.) and Alexa Fluor 488-conjugated anti-rabbit IgG (1:500, ab150077; Abcam) antibodies at room temperature for $1 \mathrm{~h}$, then stained with DAPI at room temperature for $10 \mathrm{~min}$ prior to fluorescence microscopy.

$R N A$ isolation and reverse transcription-quantitative polymerase chain reaction ( $R T-q P C R)$ analysis. NRK-49F cells were treated with or without baicalin/baicalein $(20-80 \mu \mathrm{M})$ for various periods of time (24-96 h). Total RNA was isolated using TRIzol reagent (Thermo Fisher Scientific, Inc.) according to the manufacturer's protocol. First-Strand complementary DNA was then synthesized from the total RNA by reverse transcription using the GoScript ${ }^{\mathrm{TM}}$ Reverse Transcription system (Promega Corp., Madison, WI, USA) according to the manufacturer's protocol. qPCR was conducted using TaqMan ${ }^{\circledR}$ Gene Expression assays (TGF- $\beta 1$, Rn00572010_m1; COL1A1, Rn01463848_m1; COL1A2, Rn00584426_m1; Thermo Fisher Scientific, Inc.) and a 7900HT Fast Real-Time PCR system (Thermo Fisher Scientific, Inc.) according to the manufacturer's protocol, in order to quantify rat collagen type I $\alpha 1$ (COLIA1) and 2 (COLIA2) mRNA expression. The thermocycling conditions were as follows: Initial denaturation at $95^{\circ} \mathrm{C}$ for $10 \mathrm{~min}$; followed by 40 cycles of denaturation at $95^{\circ} \mathrm{C}$ for $10 \mathrm{sec}, 60^{\circ} \mathrm{C}$ for $15 \mathrm{sec}$ and $72^{\circ} \mathrm{C}$ for $15 \mathrm{sec}$. GAPDH was used as the reference gene. Relative quantification of gene expression was performed using the $2^{-\Delta \Delta \mathrm{Cq}}$ method.

Western blotting. After a $48 \mathrm{~h}$ treatment with baicalin/baicalein $(20-80 \mu \mathrm{M})$, total protein was extracted from NRK-49F cell lysate and the protein concentration was determined using the bicinchoninic acid assay. Protein samples $(20 \mu \mathrm{g})$ were loaded and separated on a $10 \%$ gel using SDS-PAGE, and then electrotransferred to nitrocellulose membranes. After blocking with $5 \%$ nonfat milk at room temperature for $1 \mathrm{~h}$, the membranes were incubated with rabbit anti-rat polyclonal COL1 primary (1:1,000, ab34710; Abcam) and mouse anti-rat monoclonal GAPDH (1:1,000, MAB374; Merck KGaA) primary antibodies overnight at $4^{\circ} \mathrm{C}$, followed by incubation with a secondary antibody (mouse anti-rabbit IgG-HRP, sc-2357-CM, and chicken anti-mouse IgG-HRP (sc-2954; Santa Cruz Biotechnology, Inc., Dallas, TX, USA) at 1:5,000 dilutions for $1 \mathrm{~h}$ at room temperature. Protein bands were then detected using SignalFire ECL Reagent (Cell Signaling Technology, Inc., Danvers, MA, USA).

Secreted embryonic alkaline phosphatase (SEAP) reporter gene assay. HEK 293 cells (obtained from National Infrastructure of Cell Line Resource, Beijing, China) were seeded in a 96-well plate at a density of 5,000 cells per well and transfected with the SMAD-binding element (SBE)-SEAP plasmid using 


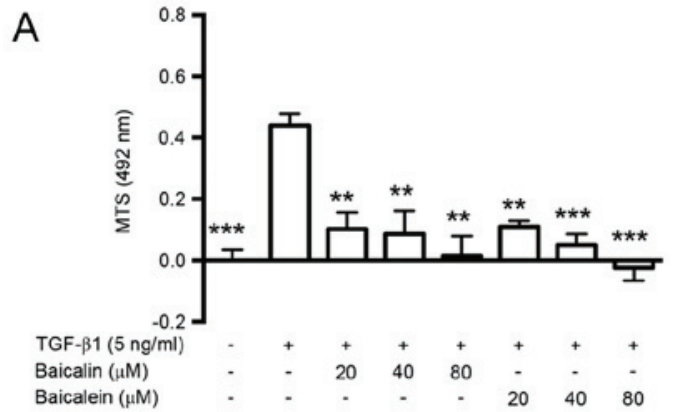

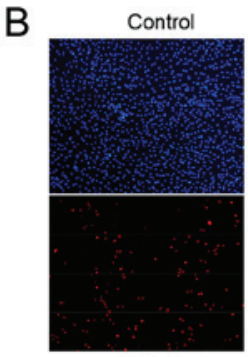

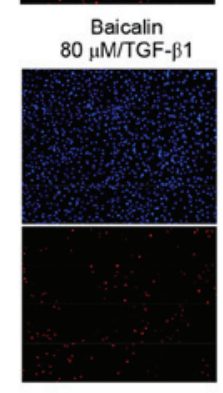

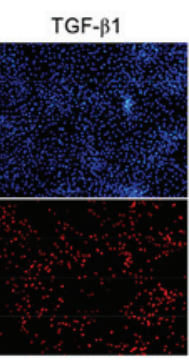

Baicalein $20 \mu \mathrm{M} / \mathrm{TGF}-\beta 1$

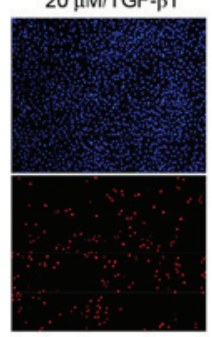

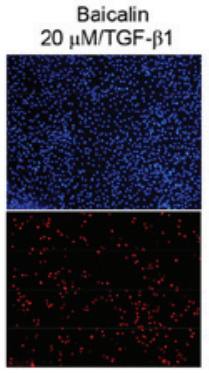

Baicalein $40 \mu \mathrm{M} / \mathrm{TGF}-\beta 1$

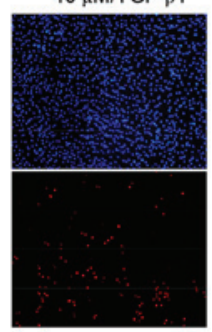

D
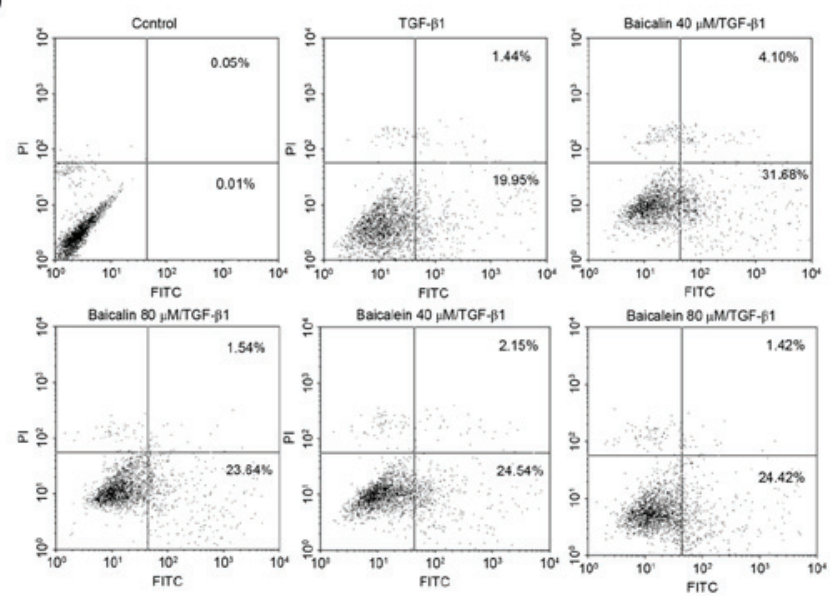

Figure 1. Baicalin and baicalein inhibit NRK-49F cell viability. Cells cultured in collagen I-coated plates were treated with $20-80 \mu \mathrm{M}$ baicalin or baicalein and exposed to $5 \mathrm{ng} / \mathrm{ml}$ TGF- $\beta 1$ for $48 \mathrm{~h}$. (A) Cells were then incubated with MTS solution and the optical density of the wells was measured at $492 \mathrm{~nm}$ after a $3 \mathrm{~h}$ incubation. BrdU was added prior to the end of experiment and immunofluorescence staining was performed to visualize BrdU incorporation. (B) Representative images of BrdU immunofluorescence staining and (C) quantification of the percentage of BrdU positive cells. (D) NRK-49F cells were stained with Annexin V/PI for 15 min and then analyzed by flow cytometry for Annexin V-positive cells. ${ }^{*} \mathrm{P}<0.05,{ }^{* *} \mathrm{P}<0.01,{ }^{* * *} \mathrm{P}<0.001$ vs. TGF- $\beta 1$ treatment alone. TGF- $\beta 1$, transforming growth factor $\beta 1$; BrdU, 5-bromo-2-deoxyuridine; I, propidium iodide; FITC, fluorescein isothiocyanate.

Fugene HD transfection reagent (Promega Corp.), as described previously (19), and the pSEAP basic plasmid as a negative control (both from Clontech Laboratories, Inc., Mountainview, CA, USA) prior to treatment with TGF- $\beta 1$ and baicalin/baicalein. The SEAP signal was quantified using the SensoLyte ${ }^{\circledR}$ p-nitrophenyl phosphate (pNPP) SEAP Reporter Gene assay kit (AnaSpec, Fremont, CA, USA) according to the manufacturer's protocol. Briefly, after treatment with baicalin/baicalein (20-80 $\mu \mathrm{M}$ ) for $48 \mathrm{~h}$, cell supernatant was collected and heated at $65^{\circ} \mathrm{C}$ for $30 \mathrm{~min}$ to inactivate non-specific alkaline phosphatase activity. The assay buffer was then added and the mixture was incubated for $5 \mathrm{~min}$ at room temperature. Subsequently, pNPP substrate buffer was added and the mixture was incubated for $1 \mathrm{~h}$ at room temperature. OD values were then measured at $405 \mathrm{~nm}$.

Statistical analysis. All data are presented as the mean \pm SEM. The statistical significances of differences between groups were analyzed via one-way analysis of the variance followed by a post hoc Newman-Keuls test. $\mathrm{P}<0.05$ was considered to indicate a statistically significant difference. All analyses were performed using GraphPad Prism software (version 6.0; GraphPad Software, Inc., La Jolla, CA, USA).

\section{Results}

Baicalin and baicalein inhibit NRK-49F cell proliferation. The effects of baicalin and baicalein on NRK-49F cell viability were investigated using the MTS and BrdU incorporation assays. In the MTS assay, TGF- $\beta 1$ significantly increased NRK-49F cell viability compared with the control group $(\mathrm{P}<0.001)$, while baicalin and baicalein dose-dependently inhibited this TGF- $\beta 1$-induced increase in cell viability at concentrations ranging from $20-80 \mu \mathrm{M}$ (Fig. 1A). The results of the BrdU incorporation assay confirmed that TGF- $\beta 1$ promoted DNA synthesis in NRK-49F cells, whereas baicalin and baicalein 
A

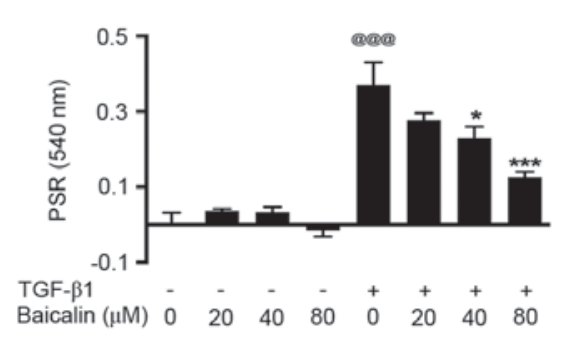

B

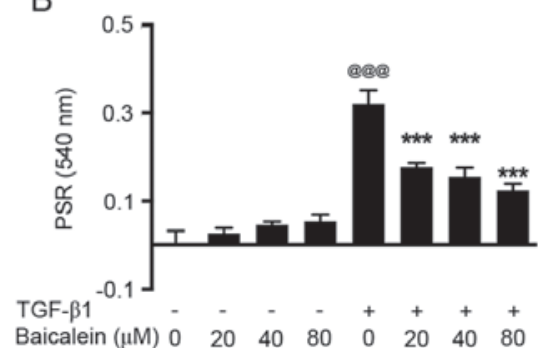

G

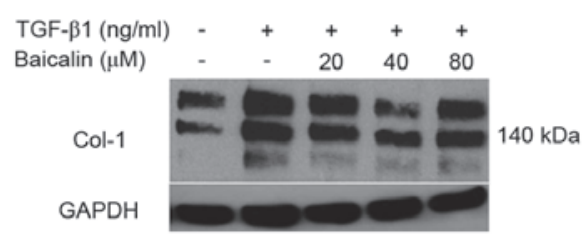

$\mathrm{H}$

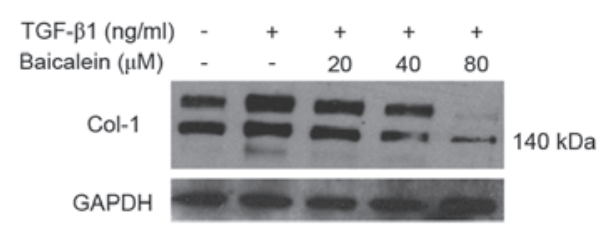

E

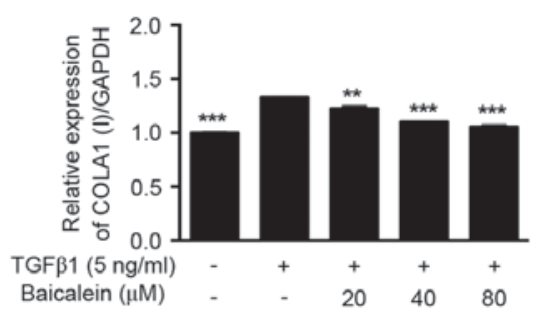

Figure 2. Baicalin and baicalein suppress the activation of NRK-49F cells and downregulate COLI expression. Cells cultured in COLI-coated plates were treated with $20-80 \mu \mathrm{M}$ (A) baicalin or (B) baicalein with or without TGF- $\beta 1$ for $48 \mathrm{~h}$. Total collagen accumulation was visualized by PSR staining and quantified by spectrophotometric analysis. Treated cells were also collected for reverse transcription-quantitative polymerase chain reaction analysis of COLA1 expression after (C) baicalin or (D) baicalein treatment and COLA2 expression after (E) baicalin or (F) baicalein treatment. The protein expression of COLI $(\sim 140 \mathrm{kDa})$ was analyzed by western blotting after $(\mathrm{G})$ baicalin or $(\mathrm{H})$ baicalein treatment. ${ }^{*} \mathrm{P}<0.05,{ }^{* *} \mathrm{P}<0.01,{ }^{* * *} \mathrm{P}<0.001 \mathrm{vs}$. TGF- $\beta 1$ treatment alone; ${ }^{@ @ @ ~} \mathrm{P}<0.001$ vs. the no treatment group. PSR, Picrosirius red; TGF- $\beta 1$, transforming growth factor $\beta 1$; COLI, collagen type I; COLA1, COL1 $\alpha 1$; COLA2, COL1 $\alpha 2$.

significantly reduced the percentage of BrdU-positive cells (Fig. 1B and C).

An Annexin V/PI double staining assay was performed to investigate the effects of baicalin and baicalein on NRK-49F cell apoptosis (Fig. 1D). Compared with the control group, the percentage of early apoptotic cells increased after $48 \mathrm{~h}$ of treatment with TGF- $\beta 1$. When the cells were treated with baicalin or baicalein $(40$ or $80 \mu \mathrm{M})$, there was no notable increase in the number of Annexin V-positive cells. These data suggest that baicalin and baicalein inhibit NRK-49F cell proliferation without inducing notable cell apoptosis.

Baicalin and baicalein inhibit TGF-ß1-induced NRK-49F cell activation. The accumulation of extracellular matrix material is considered a key event in the pathogenesis of renal fibrosis. To investigate the potential role of baicalin and baicalein in the process of TGF- $\beta 1$-induced NRK-49F cell activation, cells were activated with TGF- $\beta 1$ and stained with PSR to analyze total COLI deposition. The COLI protein accumulation induced by TGF- $\beta 1$ was significantly downregulated after baicalin (40-80 $\mu \mathrm{M}$; $\mathrm{P}<0.05$; Fig. 2A) or baicalein (20-80 $\mu \mathrm{M}$; $\mathrm{P}<0.001$; Fig. 2B) treatment compared with the control group. By contrast, baicalin and baicalein caused no effect on COLI deposition in normal unactivated NRK-49F cells (Fig. 2A and B), suggesting that they specifically antagonized TGF- $\beta 1$ activation. In addition, the mRNA and protein expression of COLI was analyzed by RT-qPCR analysis and western blotting, respectively. The expression levels of COLIA1 and COLIA2 mRNA were significantly increased after TGF- $\beta 1$ treatment $(\mathrm{P}<0.001)$; however, their expression was significantly inhibited by baicalin (40-80 $\mu \mathrm{M} ; \mathrm{P}<0.01$; Fig. $2 \mathrm{C}$ and $\mathrm{D})$ and baicalein (20-80 $\mu \mathrm{M} ; \mathrm{P}<0.001$; Fig. $2 \mathrm{E}$ and F) in a dose-dependent manner. The western blotting analysis confirmed that the upregulated expression of COLI protein $(\sim 140 \mathrm{kDa})$ following TGF- $\beta 1$ activation was inhibited by baicalin (Fig. $2 \mathrm{G}$ ) and baicalein (Fig. 2H).

Baicalin and baicalein suppress endogenous TGF- $\beta 1$ expression and activation of SMAD3. The TGF- $\beta 1$ signaling pathway is considered to be a major signaling pathway in renal fibrosis. In the present study, the endogenous expression of TGF- $\beta 1$ mRNA by NRK-49F cells was quantified by RT-qPCR analysis (Fig. 3A-D). The results revealed that the endogenous expression of TGF- $\beta 1$ by NRK-49F cells increased 
A

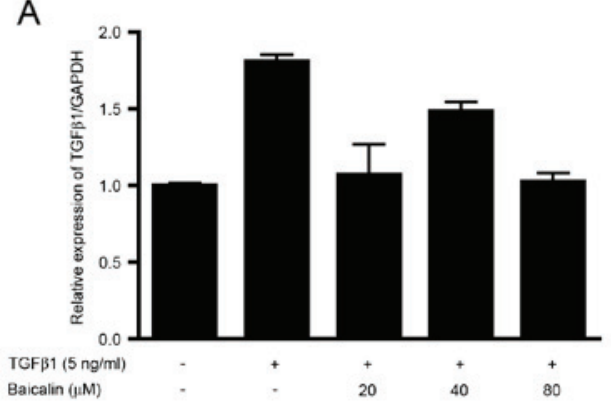

C

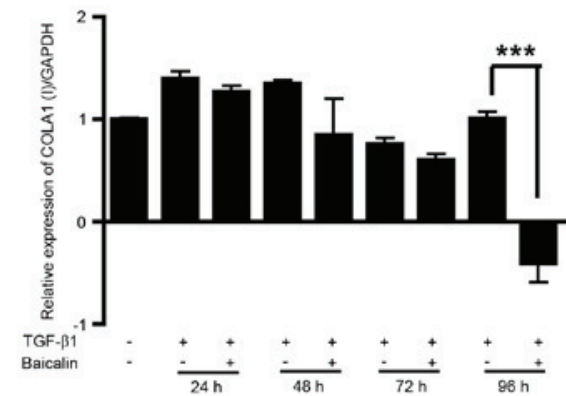

B

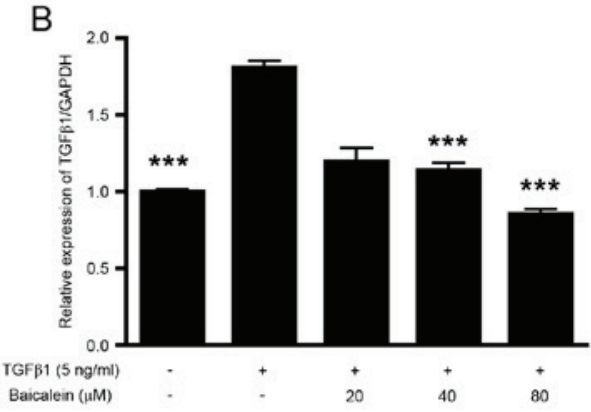

D

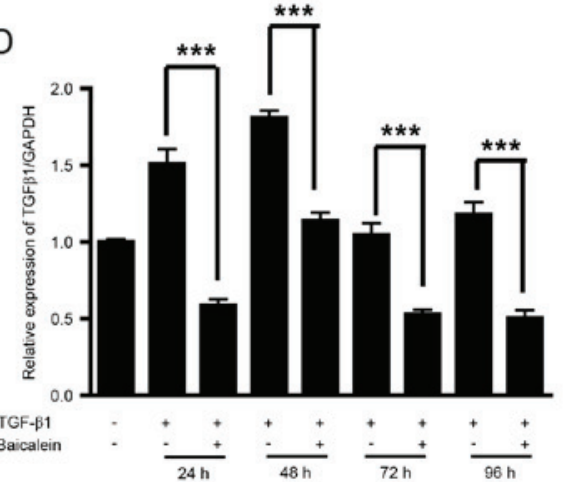

$E$
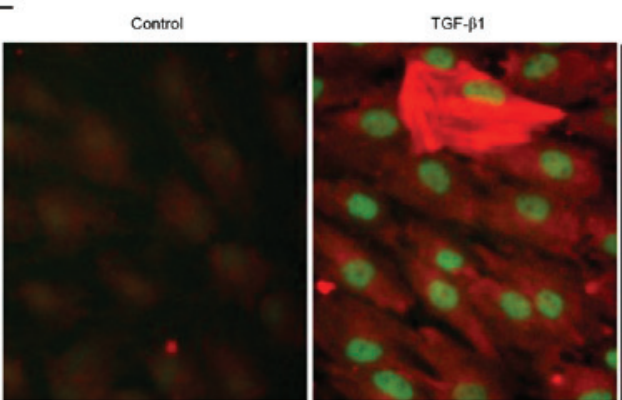

Baicalin 20 HMTGG-B1

Baicalin 40 HMTTGF- $\beta$.
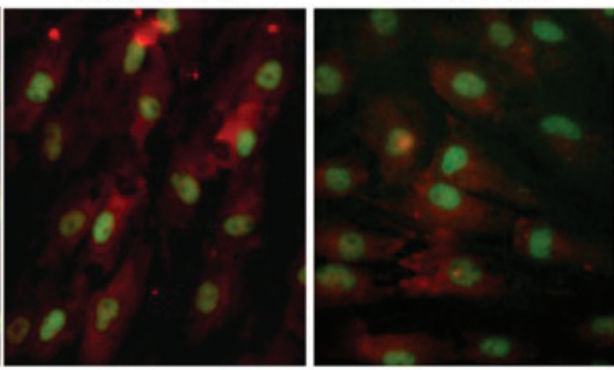

Bacain $80 \mu \mathrm{MTTF}-\beta 1$

Baicalein $20 \mu M / T G F-\beta 1$

Baicalein $40 \mu M T G F-\beta 1$
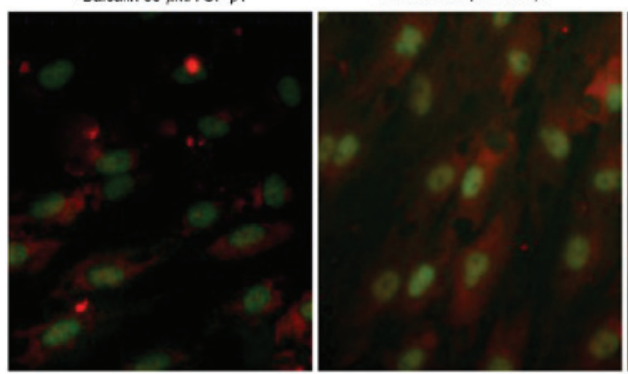

$\mathrm{F}$

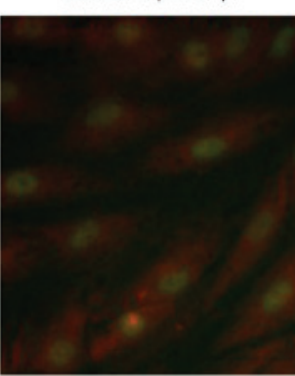

Baicalein 80 „MTTGF-B1

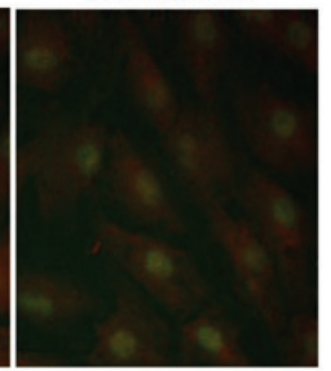

G

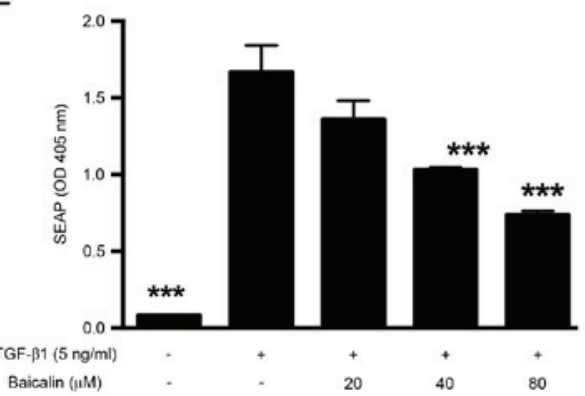

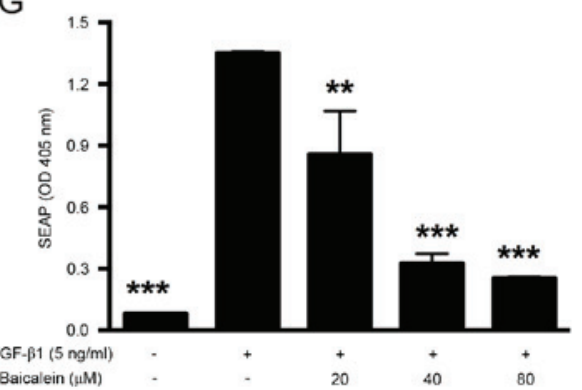

Figure 3. Baicalin and baicalein downregulate TGF- $\beta 1$-induced endogenous TGF- $\beta 1$ expression and SMAD3 activation. Cells cultured in COLI-coated plates were treated with 20-80 $\mu \mathrm{M}$ (A) baicalin or (B) baicalein. After $48 \mathrm{~h}$, cells were collected for RT-qPCR analysis of TGF- $\beta 1$ mRNA expression. Cells were treated with $80 \mu \mathrm{M}(\mathrm{C})$ baicalin or (D) baicalein and collected at 24, 48, 72 and $96 \mathrm{~h}$ for RT-qPCR analysis of TGF- $\beta 1 \mathrm{mRNA}$ expression. (E) After $48 \mathrm{~h}$ treatment with $20-80 \mu \mathrm{M}$ baicalin or baicalein double immunofluorescence staining was performed to visualize the expression of $\alpha$-SMA (red) and phosphorylated-SMAD3 (green). HEK-293T cells were transfected with the pSMAD-binding element-SEAP plasmid and then treated with $20-80 \mu$ M (F) baicalin or (G) baicalein with TGF- $\beta 1$. After $48 \mathrm{~h}$ treatment, supernatant was collected and analyzed for SEAP expression. ${ }^{* *} \mathrm{P}<0.01,{ }^{* * *} \mathrm{P}<0.001 \mathrm{vs}$. TGF- $\beta 1$ treatment alone. TGF- $\beta 1$, transforming growth factor $\beta 1$; SMAD3, mothers against decapentaplegic homolog 3; RT-qPCR, reverse transcription-quantitative polymerase chain reaction; SEAP, secreted embryonic alkaline phosphatase; COLA1, collagen type I $\alpha 1 ; \alpha$-SMA, $\alpha$ smooth muscle actin. 
after TGF- $\beta 1$ treatment, peaking at $48 \mathrm{~h}$ (Fig. 3D). Baicalein dose-dependently (40-80 $\mu \mathrm{M}$; Fig. 3B) and time-dependently (24-96 h; Fig. 3D) inhibited the endogenous expression of TGF- $\beta 1$, whereas baicalin caused weaker, but still notable, downregulation (Fig. 3A and C). The activation of SMAD3 protein was also investigated by immunostaining (Fig. 3E) and the SEAP reporter gene assay (Fig. $3 F$ and $G$ ). In the immunostaining assay, higher phosphorylated SMAD3 and $\alpha$-SMA signaling was observed after TGF- $\beta 1$ activation. The activated SMAD3 protein was accumulated in the cell nucleus and was reduced dose-dependently by treatment with baicalin or baicalein. In the SEAP reporter gene assay, the SBE-SEAP plasmid was transfected into HEK293 cells prior to treatment with TGF- $\beta 1$ and baicalin/baicalein. TGF- $\beta 1$ significantly induced SEAP reporter activity, whereas baicalin $(40-80 \mu \mathrm{m}$; $\mathrm{P}<0.001$; Fig. $3 \mathrm{~F})$ and baicalein $(20-80 \mu \mathrm{m} ; \mathrm{P}<0.01$; Fig. $3 \mathrm{G})$ significantly antagonized the SMAD3 signaling pathway in a dose-dependent manner.

\section{Discussion}

The present study investigated the antifibrotic effects and potential mechanism of action of baicalin and baicalein in an in vitro TGF- $\beta 1$-induced renal fibrosis model. The results revealed that TGF- $\beta 1(5 \mathrm{ng} / \mathrm{ml})$ could stimulate the proliferation and apoptosis of NRK-49F cells, which is consistent with previous reports $(20,21)$. It is well recognized that activated resident fibroblasts contribute to myofibroblast production in the fibrotic pathological process. A previous study by our group developed a high-throughput assay for the detection of inflammation-independent antifibrotic effects, and identified baicalin and baicalein as potential antifibrotic agents using NRK-49F cells (18). In the present study, the effects of baicalin and baicalein on TGF- $\beta 1$-activated fibroblasts and normal fibroblasts were further examined. As expected, in the presence of TGF- $\beta 1$ the total collagen accumulation, as assessed by PSR staining and the expression of COLI, the major type of collagen produced by NRK-49F cells, was enhanced and dose-dependently antagonized by treatment with baicalin or baicalein. However, basal ECM expression was not affected by baicalin or baicalein, particularly the latter. Thus, these data suggest that baicalin and baicalein specifically inhibit TGF- $\beta 1$-induced ECM accumulation.

To further explore the mechanism of action of baicalin and baicalein, endogenous TGF- $\beta 1$ expression was examined by RT-qPCR, and the activation of SMAD3 was detected by immunostaining and the SEAP gene reporter assay. Previous studies have demonstrated that TGF- $\beta$ serves an important role in fibrosis via modulation of SMAD-dependent and independent pathways $(22,23)$. SMAD-dependent signaling is initiated by the binding of TGF- $\beta 1$ to its receptor to form a heterotrimer complex, which recruits and phosphorylates SMAD2 and SMAD3 proteins. SMAD2/3 then forms heterocomplexes with the SMAD4 protein and translocates into the nucleus to regulate gene expression (24). In the present study, the SBE-SEAP plasmid, which contains a TGF- $\beta$-responsive $\mathrm{SBE}$, was used for the SEAP gene reporter assay. Previous data have demonstrated that when the SMAD3/4 complex enters the nucleus, SMAD3 directly binds to SBE elements, which contain CAGA boxes, and induces the production of
SEAP (19). SEAP can be secreted into the cell supernatant and easily assessed by various methods. However, no binding has been observed between the CAGA box and SMAD1/2, a major component in the bone morphogenetic protein signaling pathway. When the SBE-SEAP plasmid was transfected into the cells, dose-dependent inhibition of the SEAP activity was observed after baicalin and baicalein treatment, suggesting that the effects of baicalin and baicalein are TGF- $\beta 1$-specific. This data was consistent with the results obtained by immunostaining for the phosphorylated-SMAD3 protein.

Baicalin and baicalein are major bioactive flavones derived from S. baicalensis Georgi. Baicalin, the glucuronide of baicalein, is metabolized by the enteric microbiome into baicalein. In the present study baicalein demonstrated a higher activity in all the assays compared with baicalin. Previous studies have also revealed that baicalein exerted higher inhibitory effects compared with baicalin in regards to inflammation $(25,26)$, angiogenesis (27) and cancer (28). Previous metabolic and pharmacokinetic analyses have indicated that baicalin is absorbed to a lesser extent and more slowly compared with baicalein in vivo $(29,30)$. These results indicate that the presence of a glucosyl unit may contribute to the weaker activity and metabolism of baicalin.

In conclusion, the present study demonstrated that baicalin and baicalein exert dose-dependent antifibrotic effects via inhibiting TGF- $\beta 1$ expression and the activation of SMAD3. The applications and more detailed mechanisms of baicalin and baicalein merit further study.

\section{Acknowledgements}

The present study was supported by Beijing Municipal Natural Science Foundation (grant no. 7142013) and the Scientific Research Project of Beijing Educational Committee (grant no. KM201510005029). The authors would like to thank Dr Qinhe Xu and Dr Jie Jiang of King's College London (London, UK) for their help and support in conducting the experiments.

\section{References}

1. O'Dwyer DN, Ashley SL and Moore BB: Influences of innate immunity, autophagy, and fibroblast activation in the pathogenesis of lung fibrosis. Am J Physiol Lung Cell Mol Physiol 311: L590-L601, 2016.

2. Leaf IA and Duffield JS: What can target kidney fibrosis? Nephrol Dial Transplant 32 (Suppl 1): i89-i97, 2017.

3. Duffield JS: Cellular and molecular mechanisms in kidney fibrosis. J Clin Invest 124: 2299-2306, 2014

4. Meng XM, Nikolic-Paterson DJ and Lan HY: TGF- $\beta$ : The master regulator of fibrosis. Nat Rev Nephrol 12: 325-338, 2016.

5. de Oliveira MR, Nabavi SF, Habtemariam S, Erdogan Orhan I, Daglia $M$ and Nabavi SM: The effects of baicalein and baicalin on mitochondrial function and dynamics: A review. Pharmacol Res 100: 296-308, 2015

6. Xue Y, Shui X, Su W, He Y, Lu X, Zhang Y, Yan G, Huang S, Lei $\mathrm{W}$ and Chen $\mathrm{C}$ : Baicalin inhibits inflammation and attenuates myocardial ischaemic injury by aryl hydrocarbon receptor. J Pharm Pharmacol 67: 1756-1764, 2015.

7. Zhou YJ, Wang H, Sui HH,Li L, Zhou CL and Huang JJ: Inhibitory effect of baicalin on allergic response in ovalbumin-induced allergic rhinitis guinea pigs and lipopolysaccharide-stimulated human mast cells. Inflamm Res 65: 603-612, 2016.

8. Kwak S, Ku SK, Han MS and Bae JS: Vascular barrier protective effects of baicalin, baicalein and wogonin in vitro and in vivo. Toxicol Appl Pharmacol 281: 30-38, 2014. 
9. Zhu W, Jin Z, Yu J, Liang J, Yang Q, Li F, Shi X, Zhu X and Zhang X: Baicalin ameliorates experimental inflammatory bowel disease through polarization of macrophages to an M2 phenotype. Int Immunopharmacol 35: 119-126, 2016.

10. Zhao F, Fu L, Yang W, Dong Y, Yang J, Sun S and Hou Y: Cardioprotective effects of baicalein on heart failure via modulation of $\mathrm{Ca}(2+)$ handling proteins in vivo and in vitro. Life Sci 145: 213-223, 2016.

11. Yan WJ, Ma XC, Gao XY, Xue XH and Zhang SQ: Latest research progress in the correlation between baicalein and breast cancer invasion and metastasis. Mol Clin Oncol 4: 472-476, 2016.

12. Xiao JR, Do CW and To CH: Potential therapeutic effects of baicalein, baicalin, and wogonin in ocular disorders. J Ocul Pharmacol Ther 30: 605-614, 2014.

13. Huang X, He Y, Chen Y, Wu P, Gui D, Cai H, Chen A, Chen M, Dai C, Yao D and Wang L: Baicalin attenuates bleomycin-induced pulmonary fibrosis via adenosine A2a receptor related TGF- $\beta 1$-induced ERK1/2 signaling pathway. BMC Pulm Med 16: 132, 2016.

14. Peng XD, Dai LL, Huang CQ, He CM and Chen LJ: Correlation between anti-fibrotic effect of baicalin and serum cytokines in rat hepatic fibrosis. World J Gastroenterol 15: 4720-4725, 2009.

15. Wang W, Zhou PH, Xu CG, Zhou XJ, Hu W and Zhang J: Baicalein attenuates renal fibrosis by inhibiting inflammation via down-regulating NF- $\kappa$ B and MAPK signal pathways. J Mol Histol 46: 283-290, 2015.

16. Wang W, Zhou PH, Xu CG, Zhou XJ, Hu W and Zhang J: Baicalein ameliorates renal interstitial fibrosis by inducing myofibroblast apoptosis in vivo and in vitro. BJU Int 118: 145-152, 2016.

17. Liu T, Dai W, Li C, Liu F, Chen Y, Weng D and Chen J: Baicalin alleviates silica-induced lung inflammation and fibrosis by inhibiting the Th17 response in C57BL/6 mice. J Nat Prod 78 3049-3057, 2015.

18. Hu Q, Noor M, Wong YF, Hylands PJ, Simmonds MS, Xu Q, Jiang D, Hendry BM and Xu Q: In vitro anti-fibrotic activities of herbal compounds and herbs. Nephrol Dial Transplant 24: 3033-3041, 2009

19. Tesseur I, Zou K, Berber E, Zhang H and Wyss-Coray T: Highly sensitive and specific bioassay for measuring bioactive TGF-beta. BMC Cell Biol 7: 15, 2006
20. Zhang L, Zhang J, Xu C, Zhou X, Wang W, Zheng R, Hu W and Wu P: Lefty-1 alleviates TGF- $\beta 1$-induced fibroblast-myofibroblast transdifferentiation in NRK-49F cells. Drug Des Devel Ther 9: 4669-4678, 2015.

21. Zhu B, Jin Y, Han L, Chen H, Zhong F, Wang W and Chen N Proteasome inhibitor inhibits proliferation and induces apoptosis in renal interstitial fibroblasts. Pharmacol Rep 65: 1357-1365, 2013.

22. Lakshmi SP, Reddy AT and Reddy RC: Transforming growth factor $\beta$ suppresses peroxisome proliferator-activated receptor gamma expression via both SMAD binding and novel TGF- $\beta$ inhibitory elements. Biochem J 474: 1531-1546, 2017.

23. Biernacka A, Dobaczewski $M$ and Frangogiannis NG: TGF- $\beta$ signaling in fibrosis. Growth Factors 29: 196-202, 2011.

24. Meng XM, Tang PM, Li J and Lan HY: TGF- $\beta /$ Smad signaling in renal fibrosis. Front Physiol 6: 82, 2015.

25. Lee W, Ku SK and Bae JS: Anti-inflammatory effects of Baicalin, Baicalein, and Wogonin in vitro and in vivo. Inflammation 38: 110-125, 2015.

26. Nakamura N, Hayasaka S, Zhang XY, Nagaki Y, Matsumoto M, Hayasaka Y and Terasawa K: Effects of baicalin, baicalein, and wogonin on interleukin- 6 and interleukin- 8 expression, and nuclear factor-kappab binding activities induced by interleukin-1beta in human retinal pigment epithelial cell line. Exp Eye Res 77: 195-202, 2003.

27. Liu JJ, Huang TS, Cheng WF and Lu FJ: Baicalein and baicalin are potent inhibitors of angiogenesis: Inhibition of endothelial cell proliferation, migration and differentiation. Int J Cancer 106: 559-565, 2003

28. Chen J, Li Z, Chen AY, Ye X, Luo H, Rankin GO and Chen YC: Inhibitory effect of baicalin and baicalein on ovarian cancer cells. Int J Mol Sci 14: 6012-6025, 2013.

29. Miao Q, Wang Z, Zhang Y, Miao P, Zhao Y, Zhang Y and Ma S: In vitro potential modulation of baicalin and baicalein on P-glycoprotein activity and expression in Caco-2 cells and rat gut sacs. Pharm Biol 54: 1548-1556, 2016.

30. Lai MY, Hsiu SL, Tsai SY, Hou YC and Chao PD: Comparison of metabolic pharmacokinetics of baicalin and baicalein in rats. J Pharm Pharmacol 55: 205-209, 2003. 